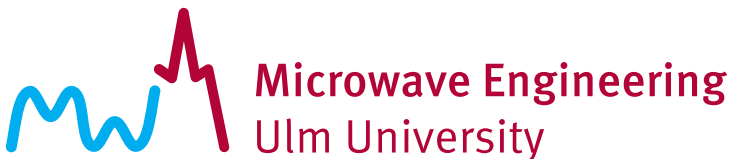

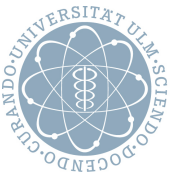

\section{Effort Considerations of Compressed Sensing for Automotive Radar}

Fabian Roos, Philipp Hügler, Jonathan Bechter, Muhammad Ahsan Razzaq, Christina Knill, Nils Appenrodt, Jürgen Dickmann, and Christian Waldschmidt

(C) 2019 IEEE. Personal use of this material is permitted. Permission from IEEE must be obtained for all other uses, in any current or future media, including reprinting/republishing this material for advertising or promotional purposes, creating new collective works, for resale or redistribution to servers or lists, or reuse of any copyrighted component of this work in other works. 


\title{
Effort Considerations of Compressed Sensing for Automotive Radar
}

\author{
Fabian Roos \#, Philipp Hügler \#, Jonathan Bechter \#, Muhammad Ahsan Razzaq \#, \\ Christina Knill *, Nils Appenrodt *, Jürgen Dickmann *, and Christian Waldschmidt * \\ \# Institute of Microwave Engineering, Ulm University, 89081 Ulm, Germany \\ *Daimler AG, Group Research and Advanced Engineering, 89081 Ulm, Germany
}

\begin{abstract}
The application of iterative compressed sensing algorithms for automotive radar is often considered as too complex for real-time evaluation. In this paper, it is shown that the number of required iterations can be chosen considerable low. To determine the necessary steps, a quality criterion is evaluated. The two examined scenarios are a reduced data rate and an interference mitigation. Measurement results are shown for different numbers of iterations to verify the sufficient reconstruction capabilities.
\end{abstract}

\section{INTRODUCTION}

The concept of compressed sensing [1], [2] was introduced in automotive radar for various tasks. Possible examples are an enhanced angular [3] or range-Doppler resolution as in [4]. In both cases samples are missing which are restored with compressed sensing algorithms.

Several algorithms have been introduced for signal reconstruction, some of them are iterative ones as the iterative method with adaptive threshold (IMAT) described in [5]. Hence, the processing effort is directly linked to the number of required iterations. To lower the processing time, an investigation is performed of how many iterations are necessary for a sufficient reconstruction. This is done using a quality criterion depending on the noise floor and signal level.

In two different measured scenarios, the iteration number dependency is shown. In one case an interferer disturbed a couple of samples leading to an enhanced noise floor. The affected data are detected and reconstructed as introduced in [6]. In the second example the data rate of the radar signal is reduced as shown in [7] and [8].

\section{Signal Processing}

The first step in the signal processing chain is to determine the positions of missing samples. In the case of data rate reduction as in [8], the positions are known due to the reduction scheme. For the interference mitigation the positions of interfered samples are not known. In literature various methods are discussed to detect interference, e.g., a simple power detector as shown in [9]. An occurring interferer has a much higher signal power than the usual baseband signal. Hence, the change in power level is exploited to detect the interference.

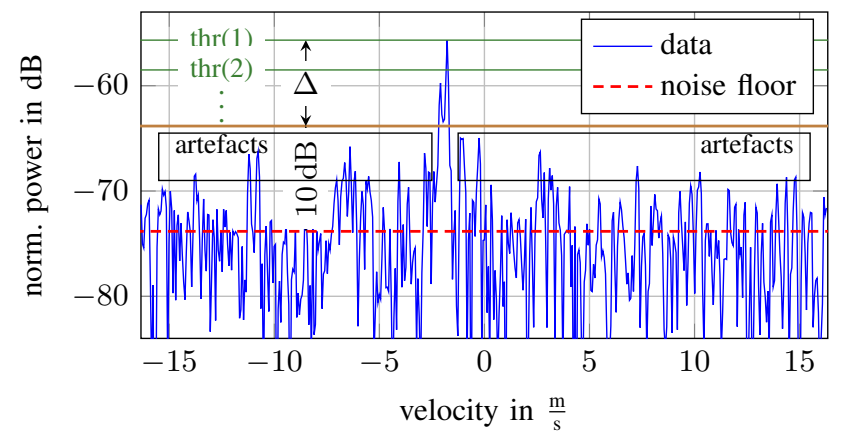

Fig. 1. Measured spectrum of one range cell if only one third of the frequency ramps are present as in [8]. Rough estimation of the noise floor (----) and the stopping level (- $10 \mathrm{~dB}$ above.

In the next step, the missing samples are reconstructed using the IMAT algorithm. As a modulation format, the fast chirp modulation [10], also known as chirp-sequence, is used. Therefore, the baseband signal is evaluated with a two-dimensional Fourier transform $(2 D-F F T)$.

\section{A. Signal Reconstruction with IMAT: Parametrisation}

The basic principle of the reconstruction algorithm is to take the incomplete signal, assume the missing samples to be zero, and calculate the Fourier transform of it. Due to the missing samples, artefacts higher than the noise floor occur in the spectrum, cf. Fig. 1. In the depicted measured spectrum of a range cell, two different frequencies are present. As a first approximation, the highest spectral occurrence is considered to be a valid frequency of the signal and used for reconstruction of the missing samples. This level is the threshold (thr), which is lowered in consecutive iterations to consider further frequencies. As already mentioned in [8], it is important to ensure that none of those artefacts are used for reconstruction as they would be intensified and considered as a target.

The noise floor (---) is estimated very roughly by taking all present samples into account. By adding a safety margin to the noise floor, it is ensured that the threshold does not reach the artefacts. In fact, the noise floor is overestimated as the possible targets are also present. This is not a drawback, as the calculation for this reason is 


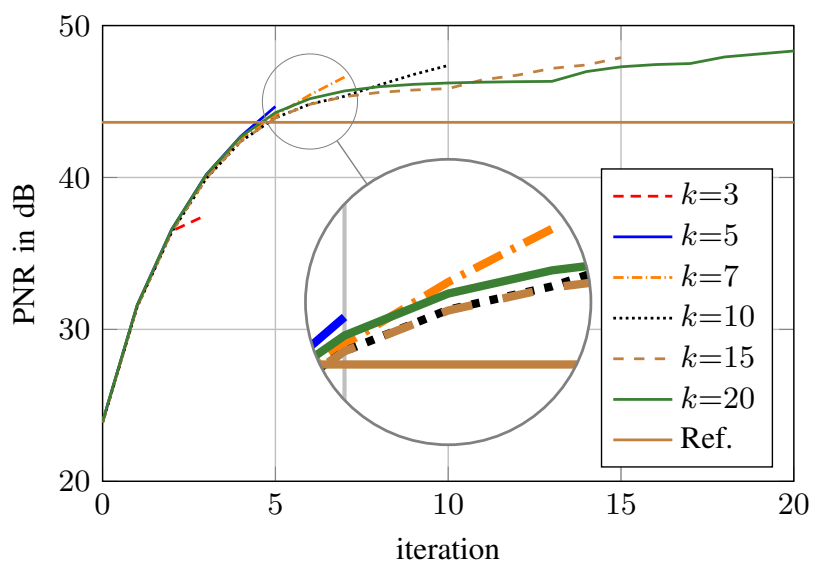

Fig. 2. Iteration number dependency of the IMAT algorithm in case of data reduction to one third with $P N R$ as quality criterion.

simple and the additional safety margin is added as well. In the case of data reduction, the amount of missing samples is known in advance, hence, the safety margin can be chosen appropriately.

Adding the safety margin to the noise floor results in the so-called stopping level ( - ), which the threshold is not allowed to exceed. With this level and the number of desired iterations, the step size of the threshold for each iteration is calculated.

\section{Simulation: Influence of Iteration Number}

As interference occurs often only for a very short time, the few affected samples do not lead to very high artefacts. In this first scenario, a single iteration is often sufficient as shown in the measurement evaluation later. Hence, the iteration number is not evaluated in simulation for interference mitigation.

Instead, the second scenario is used to show the influence of the required iteration number for a successful reconstruction. As in [8] an extended target vehicle is simulated consisting of several close scattering centres in range and velocity with different power levels. The data rate is reduced to one third and the missing samples are reconstructed afterwards. In this example, a reconstruction of over $90 \%$ of the targets is achieved in [8]. To select a more challenging scenario, complete ramps are omitted and not only random samples as this results in much higher artefact levels [8].

As a quality criterion for the reconstruction the peakto-noise ratio $(P N R)$ is chosen and shown in Fig. 2 for different iteration numbers. The artefacts are strongly influencing the noise floor, therefore, a significant change can be expected if the missing data are estimated. As the missing samples are only reconstructed with parts of the real targets, the total noise power can be lower leading to a higher $P N R$ as in the reference case (-) of a full signal. The stronger a target is, the more prominent the artefacts are in close vicinity. This is due to the fact that the artefacts originate from the convolution of the real targets with the sinc function of the missing sample pattern for a rectangular window. Therefore, the $P N R$ is shown for the strongest target.

As previously mentioned, the safety margin must be chosen such that no artefact is higher than the current threshold. A too high safety margin has the negative effect of not completely suppressed artefacts. This trade-off can be noticed for $k=3$ iterations. In this case the margin is not sufficient enough leading to fairly large threshold steps resulting in enhanced artefacts. Thus, the $P N R$ is lower after three iterations as in the other cases.

By increasing the number of iterations, the step size of the threshold is decreased. This is advantageous, as with the first iteration the influence of the missing samples is eased and weaker targets can be visible. With consecutive steps those targets are enhanced, the safety margin may be chosen smaller, and the artefacts are better suppressed.

Even in this challenging scenario an iteration number of $k=5$ is sufficient and an even higher $P N R$ as in the reference is the outcome. Spending a lot more iterations, e.g., $k=20$, the $P N R$ is increased even further, but the absolute enhancement is low compared to the effort. A reasonable iteration number is found when the $P N R$ after reconstruction is higher as for the reference signal (- $)$.

\section{Measurement Evaluation}

For verification, two measurements are conducted and for both the radar parameters are listed in Tab. I.

The interference scenario is set up in an anechoic chamber with two close targets. The interferer has a bandwidth of $300 \mathrm{MHz}$ and a different ramp duration to ensure the occurrence of interference. In Fig. 3, the interfered spectrum (---) shows an increased noise floor, which can prevent the detection of weaker targets. The interference is determined with the power detector and the zeroed samples are reconstructed with a single iteration using the IMAT algorithm (-). As only seven out of 512 samples are affected from interference, the single iteration is sufficient. Spending more iterations, e.g., 10 (--) , does not change the spectrum significantly. The advantage to reconstruct the missing samples instead of just setting them to zero is shown in [6].

TABLE I

SPECIFICATIONS OF RADAR PARAMETERS

\begin{tabular}{lrr} 
& \multicolumn{2}{c}{ Considered Scenario } \\
Parameter & Interference Mitigation & Data Reduction \\
\hline carrier frequency $f_{\mathrm{c}}$ & $76 \mathrm{GHz}$ & $76.5 \mathrm{GHz}$ \\
bandwidth $B$ & $600 \mathrm{MHz}$ & $2 \mathrm{GHz}$ \\
chirp duration $T_{\mathrm{c}}$ & $51.2 \mu \mathrm{s}$ & $50 \mu \mathrm{s}$ \\
chirp repetition time $T_{\mathrm{r}}$ & $52.5 \mu \mathrm{s}$ & $60 \mu \mathrm{s}$ \\
sampling frequency $f_{\mathrm{s}}$ & $10 \mathrm{MHz}$ & $20 \mathrm{MHz}$ \\
number of chirps $L$ & 64 & 516
\end{tabular}




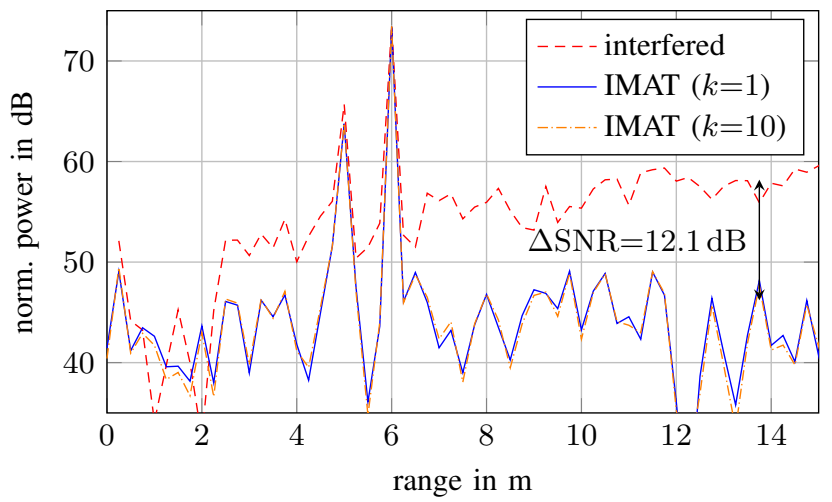

Fig. 3. Interfered (----) spectrum of a static scene with interference mitigation using two different numbers of iterations.

In case of the reduced data scenario, one third of the data are present. In Fig. 4 (a), one range cell with three different targets is shown. In contrast to the interference scenario, too many samples are missing and a single iteration (---) is not sufficient. The difference between $k=5(---)$ and $k=20$ ( $\quad$ ) is noticeable but not significant. As the simulated scenario is chosen to be in accordance with the measurement, $k=5$ iterations are selected for the reconstruction. The reconstructed range-Doppler matrix in (b) shows several reflection centres on the target vehicle and no artefacts from the missing samples. In the angle estimation result in (c) the contour of the vehicle is clearly visible. The corresponding camera image is shown in (d).

\section{CONCLUSion}

For the resource efficient application of iterative compressed sensing algorithms, it is important to determine when the reconstruction is sufficient and further iterations only lead to minor improvements. In this paper, the peakto-noise ratio is selected as a measure for a successful reconstruction and the determination of required iterations.

In the case of interference mitigation, a single iteration leads to huge improvements and is considered to be sufficient. For data reduction, where only a third of the data are present, after five iterations an artefact-free spectrum is obtained.

\section{REFERENCES}

[1] D. L. Donoho, "Compressed Sensing," Transactions on Information Theory, vol. 52, no. 4, pp. 1289-1306, Apr. 2006.

[2] E. J. Candès, J. K. Romberg, and T. Tao, "Stable Signal Recovery from Incomplete and Inaccurate Measurements," Commun. Pure Appl. Math., vol. 59, no. 8, pp. 1207-1223, Mar. 2006.

[3] F. Belfiori, et al., "Digital Beam Forming and Compressive Sensing Based DOA Estimation in MIMO Arrays," in 8th European Radar Conference, Oct. 2011, pp. 285-288.

[4] C. Knill, et al., "High Range and Doppler Resolution by Application of Compressed Sensing Using Low Baseband Bandwidth OFDM Radar," IEEE Trans. Microw. Theory Techn., vol. 66, no. 7, pp. 3535-3546, July 2018.

[5] F. Marvasti, et al., "A Unified Approach to Sparse Signal Processing," CoRR, vol. abs/0902.1853, 2009.

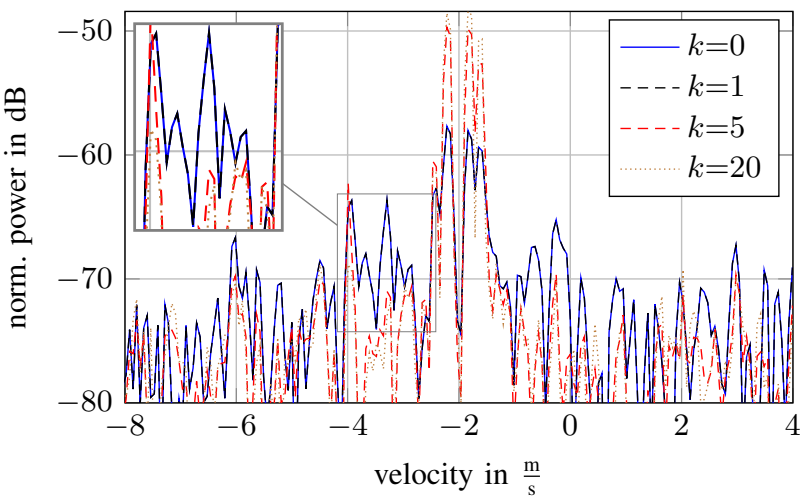

(a) The spectrum of one range cell with three targets after one iteration (-- -) is equal to the not reconstructed (- - one. No significant difference between $k=5(----)$ and $k=20(\cdots \cdots \cdots . .$.$) .$

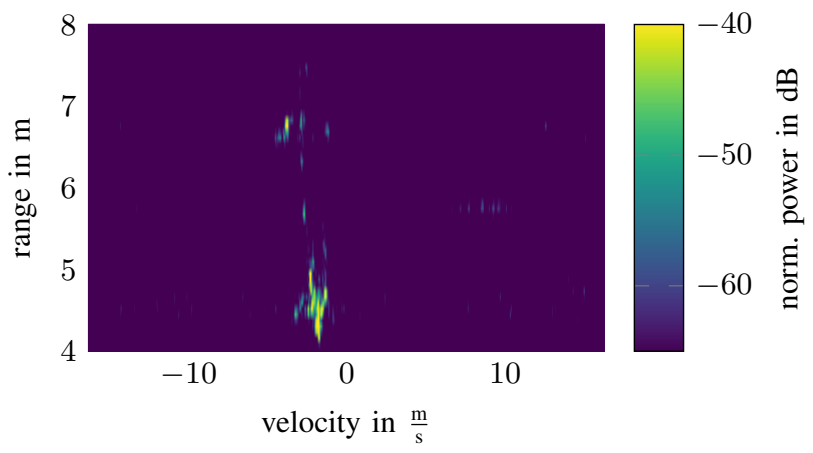

(b) Reconstructed range-Doppler evaluation after $k=5$ iterations showing different velocities of the target vehicle without noticeable artefacts.

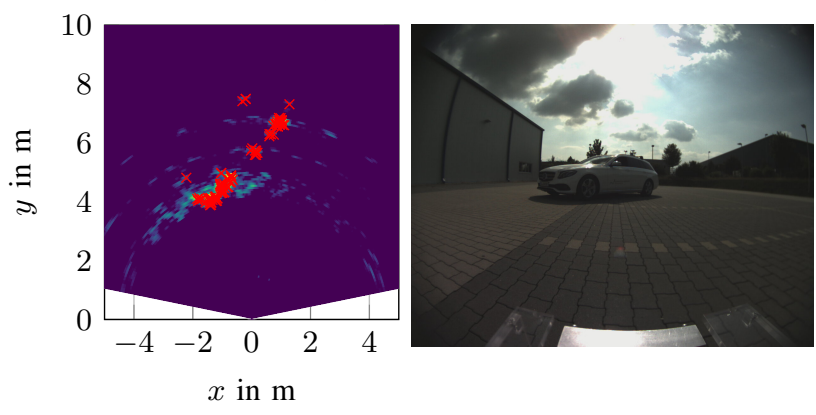

(c) After angle estimation, in the $x$ - (d) Camera image of the measured $y$ plot the contour is visible $(x)$. scene.

Fig. 4. Removal of one third of chirps and successful reconstruction.

[6] J. Bechter, et al., "Automotive Radar Interference Mitigation using a Sparse Sampling Approach," in European Radar Conference (EURAD), Oct. 2017, pp. 90-93.

[7] S. Lutz, et al., "On fast chirp Modulations and Compressed Sensing for Automotive Radar Applications," in 15th International Radar Symposium (IRS), June 2014, pp. 1-6.

[8] F. Roos, et al., "Data Rate Reduction for Chirp-Sequence based Automotive Radars using Compressed Sensing," in German Microwave Conference (GeMiC), Mar. 2018, pp. 347-350.

[9] C. Fischer, et al., "Robust Detection and Mitigation of Mutual Interference in Automotive Radar," in 16th International Radar Symposium (IRS), June 2015, pp. 143-148.

[10] V. Winkler, "Range Doppler Detection for automotive FMCW Radars," in Proceedings of the 4th European Radar Conference (EuRAD), Oct. 2007, pp. 166-169. 